\title{
The influence of albumin on the plasma xanthine oxidoreductase inhibitory activity of allopurinol, febuxostat and topiroxostat: Insight into extra-urate lowering effect
}

\author{
Takashi Nakamura $^{1 *}$, Takayo Murase ${ }^{1}$, Etsuko Satoh ${ }^{1}$, Atsushi Miyachii ${ }^{1}$, Naoyuki Ogawa ${ }^{2}$, Katsuto Abe ${ }^{2}$, Noriaki Katoh ${ }^{1}$ and Yukiharu
} Nakayama ${ }^{1}$

${ }^{1}$ Mie Research Laboratories, Sanwa Kagaku Kenkyusho Co., Ltd., Inabe-shi, Mie, 511-0406, Japan

${ }^{2}$ Clinical Development Department, Sanwa Kagaku Kenkyusho Co., Ltd., Nagoya-shi, Aichi, 461-8631, Japan

\begin{abstract}
Xanthine oxidoreductase (XOR) inhibitors, allopurinol, febuxostat, and topiroxostat are hyperuricemia and gout therapeutic drugs, which were supposed to be same. However, it was likely to be different effects in recent clinical and animal study. The activity of allopurinol is its metabolites, oxipurinol, while the others are themselves. Those XOR inhibitors have different modes of action in XOR inhibition. The XOR inhibitory activity in with or without human serum albumin (HSA) and HSA binding assay were evaluated, the constant was also calculated according to the scatchard and Klotz plot analysis. Moreover, the plasma XOR inhibitory activity was simulated based on the pharmacokinetics in repeated dose study. The XOR activity in the model increased with the $\mathrm{S} 9$ fraction. $\mathrm{IC}_{50}$ values of oxipurinol, febuxostat and topiroxostat were $244 \pm 50 \mathrm{nM}, 20.5 \pm 2.8 \mathrm{nM}$ and $1.26 \pm 0.25 \mathrm{nM}$, respectively. Febuxostat dose-dependently weakened the XOR inhibitory activity by HSA, whereas oxipurinol and topiroxostat had little impact on HSA. Additionally, the binding constant (K) to HSA in febuxostat and topiroxostat were $8.07 \times 10^{4} \mathrm{M}^{-1}$ and $1.38 \times 10^{4} \mathrm{M}^{-1}$, respectively, and the binding mode to HSA of the both drugs were nonspecific. Furthermore, XOR inhibitory activity of the lowest level in febuxostat (20 mg once a day) and topiroxostat (80mg twice a day) was estimated $61.4 \%$ and $86.8 \%$, respectively, suggesting that the dose of twice a day was desirable to serially suppress the XOR activity in the blood. Albumin impacted on plasma XOR activity of febuxostat, not topiroxostat. Therefore, topiroxostat bound highly efficient to $\mathrm{XOR}$, resulting in the strongest inhibitor of plasma XOR.
\end{abstract}

\begin{abstract}
Abbreviations: CV: Coefficient of Variation; LC: Liquid Chromatography; LLOQ: Lower Limit of Quantification; SD: Standard Deviation; RE; Relative Error; TQMS: Triple Quadrupole Mass Spectrometry; UA: Uric Acid; XDH: Xanthine Dehydrogenase; XO: Xanthine Oxidase; XOR: Xanthine Oxidoreductase; PK/PD: Pharmacokinetics/Pharmacodynamics; ALT: Alanine Transaminase; AST: Aspartate Transaminase; eGFR: estimated Glomerular Filtration Rate; HSA: Human Serum Albumin.
\end{abstract}

\section{Introduction}

Xanthine oxidoreductase (XOR) is homodimer with a molecular mass of $150 \mathrm{KDa}$ constructed from three domains, two non-identical $2 \mathrm{Fe}-2 \mathrm{~S}$ centers, one FAD cofactor and one molybdopterin cofactor, which catalyzes the oxidation of hypoxanthine to xanthine and xanthine to uric acid (UA) in the last two steps of purine metabolism in human [1,2]. As of now, allopurinol, febuxostat and topiroxostat as the XOR inhibitor (XOR-I) are used to treat hyperuricemia and gout. These XOR-Is showed not only the UA-lowering effect but also attenuating albuminuria and estimated GFR (eGFR) decline in chronic kidney disease or diabetes mellitus patients with hyperuricemia and in animal studies [3-9,10-12].

Interestingly, it seems to be some differences between XORIs. For example, Sezai et al. reported that febuxostat was superior to topiroxostat with regard to the UA reduction and antioxidant, while the renal protective and anti-inflammatory effects were similar, and Terawaki et al. reported that switching intervention from febuxostat to topiroxostat reduced proteinuria in patients with hyperuricemia $[13,14]$. Moreover, topiroxostat showed the strongest inhibitory activity in plasma even though the plasma concentration of topiroxostat was lower than that of febuxostat in animal study, suggesting that albumin contributed to the inhibitory activity [12]. Since only free drug exerts the pharmacological effect, it is important to examine the binding affinity between serum albumin and drug [15]. Furthermore, some biological characterizations of XOR-Is have been made using purified XOR enzyme. However, the examination using biological samples such as blood or organ is also essential to investigate the behavior of XOR-Is in vivo.

In the present study, to elucidate what is the cause of these differences among XOR-Is, it was verified the hypothesis that albumin could interfere with the XOR inhibitory activity by performing in vitro assay using human plasma model and human serum albumin (HSA), and was also evaluated the binding affinities between HSA and febuxostat or topiroxostat. Furthermore, according to the PK study of topiroxostat in healthy human, it was provided the insight into the plasma XOR inhibitory activity.

${ }^{\star}$ Correspondence to: Takashi Nakamura, Sanwa Kagaku Kenkyusho Co., Ltd 363 Shiosaki, Hokusei-cho, Inabe-shi Mie 511-0406, Japan, Tel: 8159472-6221, Fax: 81594820071, E-mail: ta_nakamura@mb4.skk-net.com

Key words: xanthine oxidoreductase, topiroxostat, febuxostat, allopurinol, human serum albumin, klotz plot

Received: April 29, 2019; Accepted: May 17, 2019; Published: May 21, 2019 


\section{Materials and methods}

\section{Chemicals, standards, and blank plasma}

Xanthine, hypoxanthine, and $\mathrm{NAD}^{+}$were purchased from Sigma-Aldrich Japan (Tokyo, Japan). Oxipurinol, UA, and phosphate buffered saline (PBS) were from Wako Pure Chemical Industries, Ltd. (Osaka, Japan). All other materials used were commercially available. Topiroxostat, $\left[{ }^{13} \mathrm{C},{ }^{15} \mathrm{~N}\right]$ topiroxostat, febuxostat, $\left[{ }^{2} \mathrm{H}_{6}\right]$ febuxostat, $\left[{ }^{13} \mathrm{C}_{2}\right.$, $\left.{ }^{15} \mathrm{~N}_{2}\right]$ xanthine, $\left[{ }^{13} \mathrm{C}_{2},{ }^{15} \mathrm{~N}_{2}\right] \mathrm{UA}$, and $\left[{ }^{13} \mathrm{C}_{3},{ }^{15} \mathrm{~N}_{3}\right] \mathrm{UA}$ were synthesized in our laboratory [16]. Pooled normal human plasma (EDTA-2K) and individual human plasma (EDTA-2K) were purchased from Funakoshi (Tokyo, Japan) and Tennessee Blood Services (TN, U.S.), respectively. $30 \%$ Aqueous Solution of Albumin, Human Serum (HSA) was purchased from Merck Millipore (MA, U.S.). S9 fraction in human liver homogenate was purchased from Sekisui Medical (Tokyo, Japan).

\section{Preparation of human plasma and S9}

All human plasma samples and S9 compartment were stored at $-80^{\circ} \mathrm{C}$ until each assay and were purified using Sephadex G25 column (PD-10 or PD MultiTrap G25) just before use. To prepare the plasma model with high XOR activity, S9 and human plasma were mixed at a ratio of 1:99 ( $1 \% \mathrm{~S} 9 /$ human plasma) and incubated at $37^{\circ} \mathrm{C}$ for $20 \mathrm{~min}$. Then, the mixture was used for following each assay.

\section{Model construction of high XOR activity plasma}

To establish the in vitro model of high XOR activity plasma, S9 compartment was used as a source of XOR enzyme. S9 compartment and normal human plasma were mixed at various concentrations $(0$, $0.025,0.05,0.1,0.2,0.5,1.0$ and $5.0 \% \mathrm{~S} 9$ in plasma, v/v), and XOR activity was measured as described in the section below.

\section{XOR activity assay}

XOR activity or XOR inhibitory activity were measured by using stable-isotope labeled xanthine/liquid chromatograph-triple quadrupole mass spectrometer (LC-TQMS) method [17]. In brief, the samples were mixed with PBS containing $\left[{ }^{13} \mathrm{C}_{2},{ }^{15} \mathrm{~N}_{2}\right]$ Xanthine, $\mathrm{NAD}^{+}$, and $\left[{ }^{13} \mathrm{C}_{3},{ }^{15} \mathrm{~N}_{3}\right] \mathrm{UA}$, and was incubated at $37^{\circ} \mathrm{C}$ for $90 \mathrm{~min}$. Then, $300 \mu \mathrm{L}$ methanol was added into the mixture, and was centrifuged at 3,000 $\times \mathrm{g}$ for $15 \mathrm{~min}$ at $4^{\circ} \mathrm{C}$. Subsequently, the supernatant was transferred to new tubes and dried using a centrifugal evaporator. The residues were reconstituted with $150 \mu \mathrm{L}$ distilled water, filtered through an ultrafiltration membrane, and measured using LC-TQMS which consists of a Nano Space SI-2 LC system (Shiseido, Tokyo, Japan) and a TSQ-Quantum TQMS (Thermo Fisher Scientific GmbH, Bremen, Germany) equipped with an electrospray ionization interface was used. Calibration standard sample of $\left[{ }^{13} \mathrm{C}_{2},{ }^{15} \mathrm{~N}_{2}\right]$ UA was also measured and the amounts of $\left[{ }^{13} \mathrm{C}_{2},{ }^{15} \mathrm{~N}_{2}\right]$ UA production (pmol) was quantitated from the calibration curve. XOR activity was expressed as $\left[{ }^{13} \mathrm{C}_{2},{ }^{15} \mathrm{~N}_{2}\right]$ UA in $\mathrm{pmol} / \mathrm{h} / \mathrm{mL}$ plasma. Additionally, XOR inhibitory activity (\%) were calculated by the following equation

$$
\text { Inhibitory activity }(\%)=100-\left(\frac{\left[A_{\text {XORi }}\right]}{\left[A_{\text {cont }}\right]} \times 100\right)
$$

where $\left[A_{\text {XORi }}\right]$ and $\left[A_{\text {cont }}\right]$ are the XOR activities with and without XOR-Is, respectively. IC $\mathrm{IC}_{50}$ values were calculated by Phoenix ${ }^{\circ}$ WinNonlin 6.4 software (A Certara ${ }^{\mathrm{TM}}$ Company).

\section{Electrophoresis and Western Blot analysis}

Plasma and S9 compartment samples were boiled in Laemmli sample buffer (Bio-Rad Laboratories, Inc, Hercules, CA, U.S.) for
5 min at $95^{\circ} \mathrm{C}$, and were loaded $15 \mu \mathrm{g}$ protein into the each well on the gel(Mini-PROTEAN TGX Gels 4-20\%, \#456-1095, Bio-Rad Laboratories, Inc, Hercules, CA, U.S.). Protein concentration was measured by the BCA protein assay kit (Thermo Fisher Scientific K.K. Tokyo, Japan). Then, western blotting followed by SDS-PAGE was performed, and the protein was transferred PVDF membrane (Bio-Rad Laboratories, Inc, Hercules, CA, U.S.) by Trans-BLOT TURBO (BioRad Laboratories, Inc, Hercules, CA, U.S.). Human XOR anti-body (ab109235; Abcam, Cambridge UK) and anti-Rabbit IgG, HRP (Cat\# NA934V, GE Healthcare UK Ltd.,) were used at 1:1000 and 1:5000 with $2 \%$ skim milk, respectively. The membrane was captured for 10 to 30 seconds using ECL Prime Western Blotting Detection Reagent, RPN2232 (GE Healthcare UK Ltd., Little Chalfont, Buckinghamshire, UK) by ChemiDock XRS Plus (Bio-Rad Laboratories, Inc, Hercules, CA, U.S.).

\section{Assay of XOR inhibitory activity for Oxipurinol, Febuxostat and Topiroxostat}

For the XOR inhibitory activity assay, oxipurinol, febuxostat and topiroxostat as the XOR-I were used. A $100 \mu \mathrm{L}$ of $1 \%$ S9/human plasma was mixed with $10 \mu \mathrm{L}$ of various concentrations in each XORIs, and the mixture was incubated at room temperature for $60 \mathrm{~min}$. Subsequently, XOR activities were measured as described in the above section. For this assay, one pooled and three individual plasma samples were prepared, four replicate assays were performed.

\section{Impact of HSA on XOR inhibitory activities}

To evaluate the impact of HSA on the XOR inhibitory activity, various concentrations of HSA $(0,0.4,2.0$, and $4.0 \%$ in PBS) were prepared with $S 9$ at the ratio of $99: 1$, and then, HSA in $1 \%$ S9 were mixed with various XOR-Is. The mixture was incubated at room temperature for $60 \mathrm{~min}$. Subsequently, XOR activities were measured as described in the above section. These assays were performed in duplicate.

\section{The in vitro binding affinity for HSA in febuxostat and topiroxostat}

The binding affinities between HSA and topiroxostat or febuxostat were evaluated by the ultrafiltration method. The mixture of $0.4 \%$ HSA containing $0,500,1,000,2,000,5,000,10,000$ and 20,000 ng/ $\mathrm{mL}$ of febuxostat or topiroxostat were incubated at room temperature for $5 \mathrm{~min}$. Then, the samples were added into an ultrafiltration Membrane Centrifugal device (Centrifree ${ }^{\oplus}$, Merk Millipore, MA, U.S.) and were centrifuged at $25^{\circ} \mathrm{C}$ for 20 min to collect the ultrafiltrate. Subsequently, the ultrafiltrates were mixed with $\left[{ }^{2} \mathrm{H}_{6}\right]$ febuxostat or $\left[{ }^{13} \mathrm{C},{ }^{15} \mathrm{~N}\right]$ topiroxostat as internal standard, and were measured using LC-TQMS (Nano Space SI-2/TSQ-Quantum). The standard solutions of topiroxostat or febuxostat were also measured, and the drug concentrations of the ultrafiltrate were calculated from the standard curve. These assays were performed in duplicate. The binding parameters of febuxostat or topiroxostat to HSA were estimated by the following equation for scatchard plot and double reciprocal plot (Klotz plot) [18],

$$
\frac{r}{C u}=n \cdot K-r K \text { and } \frac{1}{r}=\left(\frac{1}{n \cdot K}\right) \times \frac{1}{C u}+\frac{1}{n}
$$

where $\mathrm{Cu}$ is the concentration of the non-binding drug, $r$ the ratio of bound drug to the HSA in molar concentration, $n$ the number of binding sites on one protein molecule, and $K$ the association constant, respectively. 


\section{Simulation of the human plasma XOR activity after febuxostat and topiroxostat dosing}

To estimate the profile of the XOR inhibitory activity in plasma after febuxostat or topiroxostat dosing, pharmacodynamics analysis were performed using the drug concentrations based on the pharmacokinetic study of the repeated dose study when TMX-67(febuxostat) was orally administrated at a dose $20 \mathrm{mg}$ (in 1 dose) for 7 days in normal renal function subjects or topiroxostat was orally administered at a dose $80 \mathrm{mg}$ (in 2 doses) after the morning and evening meals for 7 days in healthy subjects, respectively $[19,20]$. The XOR inhibitory activity (E) of the drug at each time point after administration was calculated using Emax model formula [21];

$$
E(\%)=\frac{\left(E_{\max } \times C\right)}{\left(C+E C_{50}\right)}
$$

where $E_{\max }$ is the maximal effect of febuxostat and topiroxostat, and $E C_{50}\left(I C_{50}\right)$ is the concentration $(\mathrm{ng} / \mathrm{mL})$ of febuxostat and topiroxostat where $50 \%$ of its maximal effect is observed. $C$ is plasma concentration $(\mathrm{ng} / \mathrm{mL})$ of febuxostat and topiroxostat at each time point.

\section{Statistical analysis}

Data are expressed as the means \pm SD. Statistical analysis was using the statistical analysis software (SAS) program version 8.0 (SAS Institute, Cary, NC, USA), which was performed by unpaired t-test about the group of control pooled plasma and S9 mixure in the model. Multiple regression analysis was used to examine the influence of each drug and HSA concentration on XOR inhibitory activity. Less than 0.05 of P-value was regarded as statistically significant.

\section{Results}

\section{The high XOR activity model for in vitro assay}

The XOR activity increased with the $S 9$ fraction in the range of 0.025 to $5 \%$ relative to the pooled control plasma (Figure 1a). In the following assay, the mixture in this model was selected 1\% S9 for XOR inhibitory activity. Alanine transaminase (ALT) and aspartate transaminase (AST) in model plasma were significantly higher than that in control plasma (ALT; $23.8 \pm 0.5 \mathrm{U} / \mathrm{L}$ vs. $350 \pm 0.5 \mathrm{U} / \mathrm{L}(\mathrm{n}=4), \mathrm{p}<0.001$, AST; $12 \pm 1.4$ $\mathrm{U} / \mathrm{L}$ vs. $140 \pm 1.4 \mathrm{U} / \mathrm{L}(\mathrm{n}=4), \mathrm{p}<0.001)$. Additionally, XOR protein in S9 compartment was confirmed by western blotting (Figure $1 \mathrm{~b}$ ).

\section{Inhibitory effects of XOR-Is in the present model}

Plasma XOR activity in the absence of inhibitor was $2240 \pm 183$ $\mathrm{pmol} / \mathrm{h} / \mathrm{mL}(\mathrm{n}=4)$, the inhibition curves of each XOR inhibitor showed sigmoidicity (Figure 2). $\mathrm{IC}_{50}$ values and 95\% confidence interval (CI) of oxipurinol, febuxostat and topiroxostat were $244 \pm 50 \mathrm{nM}$ [95\% CI: 202-286], $20.5 \pm 2.8 \mathrm{nM}$ [95\% CI: 18.1-22.9] and 1.26 $\pm 0.25 \mathrm{nM}$ [95\% CI: 1.05-1.47] $(\mathrm{n}=4)$, respectively (Figure 2).

\section{Impact of HSA on XOR inhibitory activities}

Multiple regression analysis was performed to investigate the factor in the change of XOR inhibitory activity, and was revealed that the drug concentration in all drugs are associated with XOR inhibitory activity $(\mathrm{p}<0.0001)$ and HSA concentration was dependently associated with it of febuxostat $(\mathrm{p}=0.019)$, but oxipurinol and topiroxostat were independently associated with it $(\mathrm{p}=0.537$ and $\mathrm{p}=0.491$, respectively). Thus, as HSA concentration rose $0 \%$ to (a)

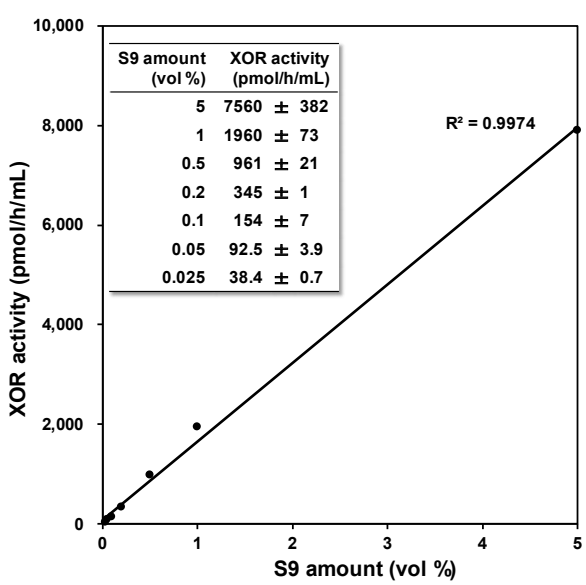

(b)

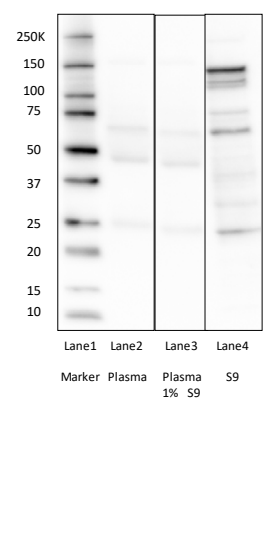

Figure 1. (a) The linearity of XOR activity with human liver S9 compartment. XOR activities $(\mathrm{pmol} / \mathrm{h} / \mathrm{mL})$ data were expressed as mean $\pm \mathrm{SD}(\mathrm{n}=3)$, (b) Protein analysis of XOR in the S9 compartment and plasma. Lane 1: molecular standard, Lane 2: Human plasma 15 $\mu \mathrm{g}$, Lane 3: Human plasma and 1\% S9 compartment 15 $\mu \mathrm{g}$, Lane4: S9 compartment $15 \mu \mathrm{g}$ on the membrane of anti-human XOR anti-body

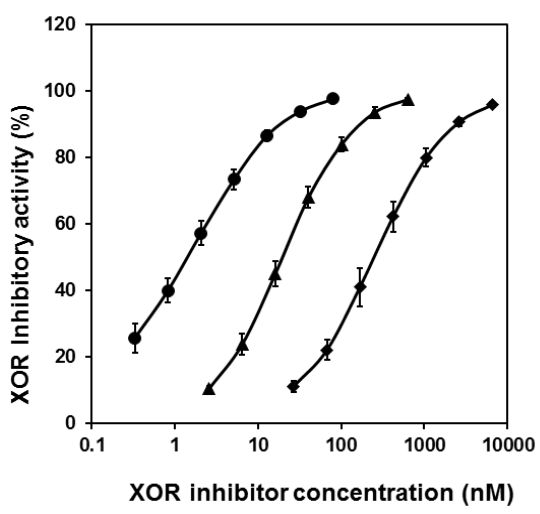

Figure 2. Inhibition curves of oxipurinol (diamonds), febuxostat (triangles) and topiroxostat (circles) on XOR activities in the mixture of human plasma and liver S9. Values of \% inhibition were expressed as mean $\pm \operatorname{SD}(n=4)$

$4 \%$, the inhibition curve in febuxostat shifted significantly to right side, whereas oxipurinol and topiroxostat were not significant with exogenous HSA concentration (Figure $3 \mathrm{a}-\mathrm{c}$ ). The $\mathrm{IC}_{50}$ values and $95 \%$ CI of oxipurinol, febuxostat and topiroxostat in the concentration of $0 \%, 0.4 \%, 2 \%$ and $4 \%$ were showed as Figure $3 \mathrm{~d}$.

\section{HSA binding assay for febuxostat and topiroxostat}

To evaluate the binding mode and affinities to HSA in febuxostat or topiroxostat, the binding parameters were estimated by the scatchard and klotz plot analysis (Figure 4a). According to the scatchard and klotz plot analysis, the $\mathrm{r} / \mathrm{Cu}$ was almost constant depending on increase in the $r$. In double reciprocal plot analysis, the slope and y-intercept of febuxostat were 6.10 and 0.492 , respectively, and their parameters of topiroxostat were 99.9 and 1.38 , respectively. Hence, in the binding constant $(\mathrm{K})$ to HSA in febuxostat and topiroxostat were $8.07 \times 10^{4} \mathrm{M}^{-1}$ and $1.38 \times 10^{4} \mathrm{M}^{-1}$, respectively, and the number of binding sites per protein molecule was 2.03 and 0.72 , respectively (Figure $4 \mathrm{~b}$ ). Binding parameters for oxipurinol could not be determined because it does not bind to albumin. 
(a)

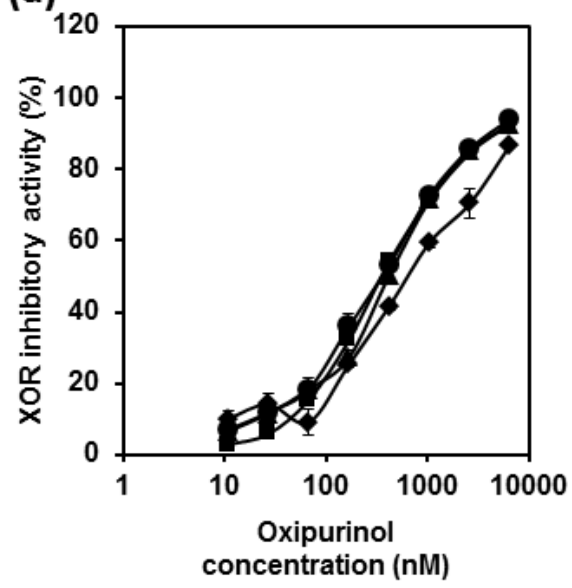

(b)

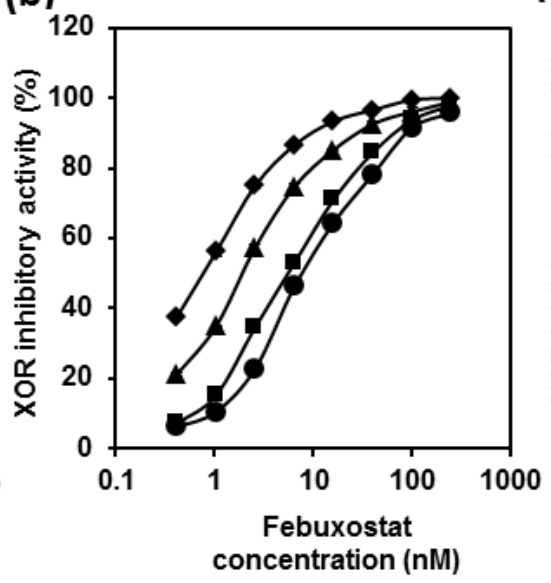

(c)

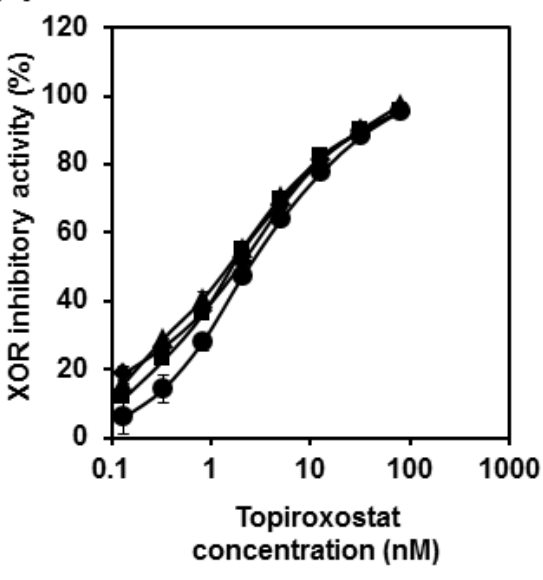

(d)

\begin{tabular}{|c|c|c|c|c|c|c|c|c|c|c|c|c|}
\hline \multirow{2}{*}{$\begin{array}{c}\mathrm{HSA}(\%) \\
\mathrm{IC}_{50}(\mathrm{nM})\end{array}$} & \multicolumn{3}{|c|}{$0 \%$} & \multicolumn{3}{|c|}{$0.4 \%$} & \multicolumn{3}{|c|}{$2.0 \%$} & \multicolumn{3}{|c|}{$4 \%$} \\
\hline & mean & $\pm \mathrm{SD}$ & $95 \% \mathrm{Cl}$ & mean & $\pm \mathrm{SD}$ & $95 \% \mathrm{Cl}$ & mean & $\pm \mathrm{SD}$ & $95 \% \mathrm{Cl}$ & mean & $\pm S D$ & $95 \% \mathrm{Cl}$ \\
\hline Oxipurinol & 461 & \pm 62 & {$[375,547]$} & 377 & \pm 40 & {$[322,432]$} & 347 & \pm 22 & {$[316,378]$} & 303 & \pm 28 & {$[264,341]$} \\
\hline Febuxostat & 0.760 & \pm 0.018 & {$[0.735,0.785]$} & 1.82 & \pm 0.06 & {$[1.74,1.90]$} & 5.45 & \pm 0.04 & {$[5.39,5.50]$} & 8.06 & \pm 0.43 & {$[7.46,8.65]$} \\
\hline Topiroxostat & 1.22 & \pm 0.03 & {$[1.18,1.26]$} & 1.03 & \pm 0.04 & {$[0.98,1.09]$} & 1.30 & \pm 0.03 & {$[1.27,1.34]$} & 2.12 & \pm 0.29 & {$[1.72,2.52]$} \\
\hline
\end{tabular}

Figure 3. Inhibition curves of oxipurinol (a), febuxostat (b) and topiroxostat (c) on XOR activities in human liver S9 with varying concentrations of HSA, (d) Summary table of IC falues $_{50}$ in each drug. Inhibition curves of $0 \%$ (diamonds), $0.4 \%$ (triangles), $2 \%$ (squares) and $4 \%$ (circles) HSA. The horizontal axes represent the final concentrations of XOR inhibitors in HSA $\mathrm{S} 9$ mixtures. Values of $\mathrm{IC}_{50}$ and $95 \%$ confidence interval (CI) were expressed as mean $\pm \mathrm{SD}$ and in parenthesis, respectively

(a)

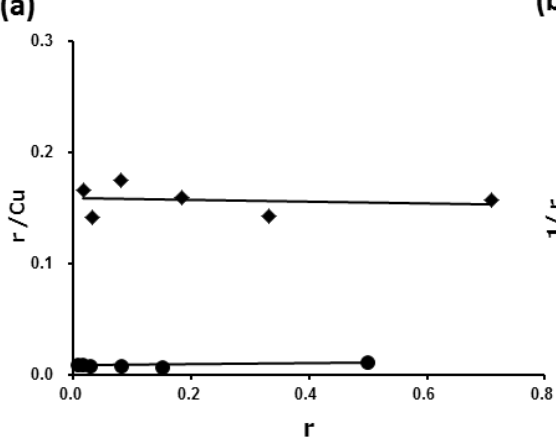

(b)

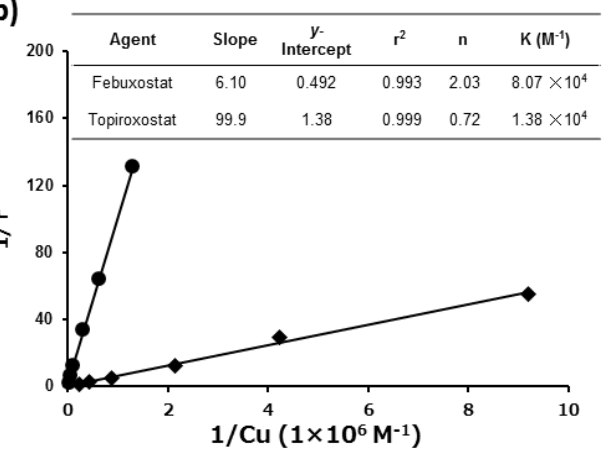

Figure 4. Scatachard plot (a) and Klotz plot (b) analysis with regard to the affinity of XOR inhibitors, febuxostat (diamonds) and topiroxostat (circles) to the HSA

\section{Simulation of the human plasma XOR activity after febuxostat and topiroxostat dosing}

Hosoya et al. reported the results of pharmacokinetic study after febuxostat dosing (Figure S1), and that of topiroxostat dosing was also shown (Figure 6a) [19,20]. Emax and $\mathrm{EC}_{50}$ values of febuxostat and topiroxostat were calculated from the inhibitory curve by in vitro models shown in Figure 2, their values were $101.1 \%$ and $6.47 \mathrm{ng} / \mathrm{mL}$ and $96.2 \%$ and $0.312 \mathrm{ng} / \mathrm{mL}$, respectively. The plasma XOR inhibitory activity at 24 hours after febuxostat dosing (10,20 and $40 \mathrm{mg}$ group) and at 12 hours after topiroxostat dosing (40,80 and $120 \mathrm{mg}$ group) were $44.1 \%, 61.4 \%, 76.4 \%$, and $79.1 \%, 86.8 \%, 89.7 \%$, respectively (Figure 6b,6c).

\section{Discussion}

In the present study, the in vitro assay model of high plasma XOR activity mimicking intravascular condition was framed. Recently, Murase et al. has reported that XOR activity in normal human plasma was low as $89.1 \pm 55.1 \mathrm{pmol} / \mathrm{h} / \mathrm{mL}(\mathrm{n}=20)$, it was too low to accurately measure the plasma XOR inhibitory activity [17]. XOR protein processed in the liver was released into the blood stream when hepatocytes turned over and was modified by protease in the blood stream [22]. This XOR life leads us to the conclusion that the mixture with commercial normal human plasma and exogenous human liver S9 compartment is an ideal assay model for the XOR inhibitory activity. It is important is that this assay model was executed using liver 


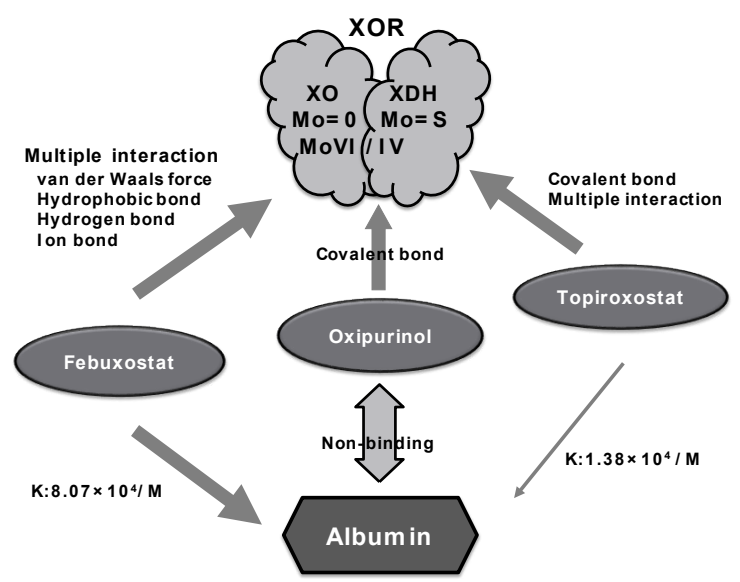

Figure 5. The summary of the interactions (binding mode to XOR and equilibrium binding constants to albumin) between XOR and albumin on the XOR inhibitors, allopurinol, febuxostat and topiroxostat

(a)

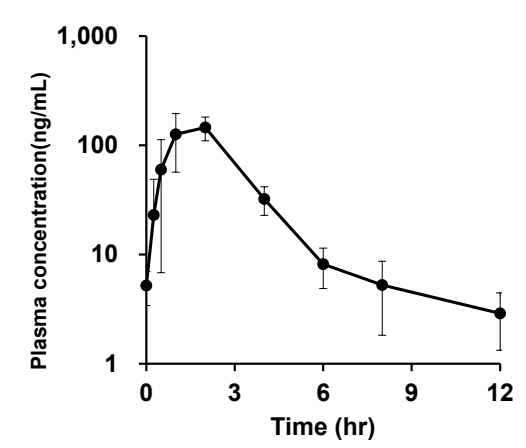

(b)

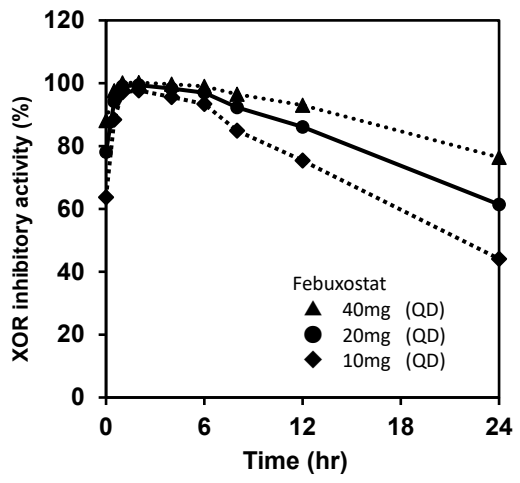

(c)

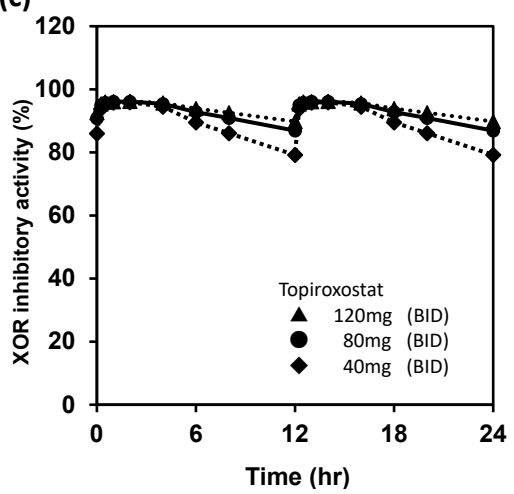

Figure 6. Plasma drug concentrations after repeated oral administration of topiroxostat in $80 \mathrm{mg}$ (BID) for 7 days in healthy human (a). The XOR inhibitory curve of febuxostat and topiroxostat shown in figure 2 to calculate the value of $\mathrm{E}_{\text {and }} \mathrm{EC}_{50}$ for $\mathrm{E}$ model analysis. The XOR inhibitory activity of febuxostat (b) and topiroxosstat (c) were simulated according to the PK data (at 0 to 12 or 24 hours) when $20 \mathrm{mg}$ (in 1 dose, circle) of febuxostat or $80 \mathrm{mg}$ (in 2 doses, circle) of topiroxostat were administrated to healthy human for 7 days in repeateddose study, respectively.; febuxostat 10mg-dose (in 1 dose, diamonds) and 40mg-dose (in 1 dose, triangle) and topiroxostat $40 \mathrm{mg}$-dose (in 2 doses, diamonds) and $120 \mathrm{mg}$-dose (in 2 doses, triangle) were proportionately calculated

homogenate, but not purified enzyme, as the XOR enzyme to be closer to the in vivo environment.

The concentration of $S 9$ compartment in the mixture was selected $1 \%$, which was enough to evaluate the XOR inhibitory activity (Figure 1a). It was approximately 25 times higher than normal plasma of previous report and was also covered the plasma XOR activity in general population of cohort study and in the patients with diabetes and chronic heart failure [17,23-25]. However, XOR protein in the mixture did not detect (Figure $1 \mathrm{~b}$ ). It might be because that the XOR protein is very sensitive and instantly converts to $\mathrm{XO}$ or is decomposed by proteolysis [26].

Allopurinol and topiroxostat showed time-dependent inhibition as described in the previous reports [27,28]. In the preliminary study, the $\mathrm{IC}_{50}$ value of oxipurinol and topiroxostat were decreased with the pre-incubation-time; nevertheless, febuxostat was not altered (Figure S2). It was because that each drug needed time to achieve in an equilibration of the binding condition in the mixture. Thus, in the present study, pre-incubation time of $60 \mathrm{~min}$ was selected for the inhibitory activity assay.

The $\mathrm{IC}_{50}$ value of topiroxostat was the lowest, and that of febuxostat and oxipurinol were 16 and 194 folds higher, respectively (Figure 2). Additionally, the XOR inhibitory activity of febuxostat was countered according to the HSA concentration, whereas that of oxipurinol and topiroxostat were insignificant (Figure 3).These results agreed with the previous report in the animal model, suggesting that albumin, which is the most abundant and transporter protein of many drugs in blood, was subjected to the interference of the interaction between febuxostat and the XOR enzyme [12,29]. Thereby, the binding mode and affinities of febuxostat and topiroxostat against HSA were evaluated.

According to the result of the scatchard and klotz plot analysis, the binding mode of both drugs to HSA were nonspecific binding (Figure $4 \mathrm{a})$, and the binding constant $(\mathrm{K})$ of febuxostat was 5.8 times higher than that of topiroxostat (Figure $4 \mathrm{~b}$ ) and the number of febuxostat binding per one albumin molecule was also 2.8 holds higher relative to that of topiroxostat. Thus, $\mathrm{IC}_{50}$ value was higher relative to topiroxostat. This result might be because that topiroxostat was easy to release from the albumin and non-binging drug has biological availability, which was remaining in the plasma, resulting in that topiroxostat exerted the inhibitory activity of XOR.

These XOR-Is have each binding mode for the molybdenum pterin center which is the active site on XOR. Allopurinol is purine analogue, whereas febuxostat and topiroxostat are non-purine selective inhibitors $[28,30]$. Oxipurinol, which is an active metabolite of allopurinol, is 
Nakamura T (2019) The influence of albumin on the plasma xanthine oxidoreductase inhibitory activity of allopurinol, febuxostat and topiroxostat: Insight into extra-urate lowering effect

bound to XOR by covalent bond, febuxostat has multiple interactions including in hydrogen-bonding, van der Waals force, hydrophobic interaction, and ion binding, and topiroxostat has both of multiple interactions and covalent bound in the mechanism (Figure 5) $[28,31,32]$. Here, allopurinol dose not bind to albumin, so the evaluation of the affinity against HSA did not be performed. Hence, this result suggests that topiroxostat may exert the inhibitory effect of plasma XOR activity at lower concentrations than febuxostat dose, and it is highly efficient.

What is to inhibit the plasma XOR activity for physiological significance. There are some people who reported that elevated plasma XOR activity was associated with obesity, diabetic mellitus, cardiovascular, and hepatic disease in human, which can produce superoxide with uric acid and the rose ROS leading to the increased risk of cardiovascular disease and mortality organ injury [29,33-43]. Moreover, Spiekermann et al. reported that post-heparin plasma was also increased plasma XO activity in patients with coronary artery disease, resulting that the tissue or organ where free XORs bound to glycosaminoglycans on the blood endothelium cells might be injured by XO-induced superoxide in the local area [38]. However, this process remains unclear. Furthermore, Eugene et al. [44] reported that the temporally reciprocal relationship between UA and nitric oxide in human circadian rhythm suggests that the inhibition of XOR activity might be serious to maintain the NO-mediated vasodilation and decrease UA level in the night phase. Thus, it was likely to be very important to inhibit the plasma XOR activity.

According to the usage of each drug, PK/PD simulation was performed to estimate the plasma XOR activity after dosing in febuxostat (20mg QD) and topiroxostat ( $80 \mathrm{mg}$ BID), and the minimum inhibitory activities (\%) was $61.4 \%$ at 24 hours and $86.8 \%$ at 12 hours after dosing, respectively (Figure 6b, 6c). In recently, Srinivasan B et. al., reported that febuxostat intervention in patients with hyperuricaemia and type 2 diabetic nephropathy did not decrease the urinary albumin, which is an injury marker of vascular endothelial cell, whereas topiroxostat did it in the randomized clinical study $[7,45]$. In the point of view of a vasoprotection, it is important to continue the inhibition of extra XOR induced ROS in the blood through a day. Therefore, it was suggested that topiroxostat can be sustainable suppression of the XOR activity in the blood owing to twice a day, resulting in the suppression of the albuminuria in the clinical trials.

There are some limitations in the present study. Firstly, XOR has complicated forms; xanthine dehydrogenase $(\mathrm{XDH})$ uses $\mathrm{NAD}^{+}$as an electron accepter, while xanthine oxidase (XO) uses oxygen to generate superoxide and $\mathrm{H}_{2} \mathrm{O}_{2}[1,46-48]$. Then, XOR is the active $(\mathrm{Mo}=\mathrm{S})$ or inactive $(\mathrm{Mo}=\mathrm{O})$ form according to an atom combined with molybdenum atom, the former is so-called "de-sulfo" type, the latter is so-colled "sulfo" type, and oxidized $(\mathrm{Mo}(\mathrm{VI}))$ or reduced $(\mathrm{Mo}(\mathrm{VI})$ ) form [26]. Additionally, it is easy to exchange the conformation by freeze-throw, preparation, etc., Thus, XOR is not the same condition in which it is in the mixture and blood stream in vivo. However, S9 fraction from liver homogenate was prepared in the presence of allopurinol [49]. Therefore, it suggested that XOR conversion, especially, to inactive form might be a minimum. Secondly, the anchoring XOR activity was not reflected in the present assay. Finally, a clinical intervention study would be necessary to ensure the result of simulation.

\section{Conclusion}

The high XOR activity model mixed with human plasma and liver S9 compartment was theorized. According to the $\mathrm{IC}_{50}$ value in XOR inhibitory curve, topiroxostat was the strongest inhibitor and albumin has little effect on the response to topiroxostat. In contrast, febuxostat was stronger than topiroxostat in HSA binding, so that XOR inhibitory activity might be weaker. Therefore, topiroxostat may exert the XOR inhibitory activity in the low concentration. This means it highly efficient. Moreover, according to the result of the simulation, topiroxostat (80mg, in 2 doses) can sequentially inhibit plasma XOR activity through a day.

\section{Author contribution}

TN and TM wrote the manuscript. TN, TM, AM, NK and YN designed the research. TM and ES performed the research and collecting data. TM, AM and YN analyzed the data. NO and KA performed the statistical analysis.

\section{Acknowledgements}

The authors would like to thank the technical assistance of Ms.Minami Inagaki, Ms.Ai Matsushita, and Ms. Hiromi Nishitani for sample measurement.

\section{Conflicts of Interest}

All authors are employees of Sanwa Kagaku Kenkyusho Co., Ltd. The authors have no conflicts of interest to disclose.

\section{Funding}

This work is an original of our own, no funds and methodology for this study receive from other company or government at all.

\section{References}

1. Amaya Y, Yamazaki K, Sato M, Nada K, Nishino T, et al. (1990) Proteolytic conversion of xanthine dehydrogenase from the NAD-dependent type to the O2-dependent type. Amino acid sequence of rat liver xanthine dehydrogenase and identification of the cleavage sites of the enzyme protein during irreversible conversion by trypsin. $J$ Biol Chem 265: 14170-14175.

2. Bray RC (1975) Molybdenum iron-sulfer Flavin hydroxylase and related enzymes. In the Enzymes XII (Boyer PD, ed), pp300-419, Academic Press, New York, San Francisco, London.

3. Goicoechea M, de Vinuesa SG, Verdalles U, Ruiz-Caro C, Ampuero J, et al. (2010) Effect of allopurinol in chronic kidney disease progression and cardiovascular risk. Clin J Am Soc Nephrol 5: 1388-1393. [Crossref]

4. Kanbay M, Ozkara A, Selcoki Y, Isik B, Turgut F, et al. (2007) Effect of treatment of hyperuricemia with allopurinol on blood pressure, creatinine clearance, and proteinuria in patients with normal renal function. Int Urol Nephrol 39: 1227-1233.

5. Michael AB, H Ralph Schumacher (2010) The urate-lowering efficacy and safety of febuxostat in the treatment of the hyperuricemia of gout: the CONFIRMS trial Arthritis Res Ther 12: R63.

6. Whelton A, Macdonald PA, Zhao L, Hunt B, Gunawardhana L (2011) Renal function in gout: long-term treatment effects of febuxostat. J Clin Rheumatol 17: 7-13. [Crossref]

7. Hosoya T, Ohno I, Nomura S, Hisatome I, Uchida S, et al. (2014) Effects of topiroxostat on the serum urate levels and urinary albumin excretion in hyperuricemic stage 3 chronic kidney disease patients with or without gout. Clin Exp Nephrol 18: 876-884. [Crossref]

8. Wada T, Hosoya T, Honda D, Sakamoto R, Narita K, et al. (2018) Uric acid-lowering and renoprotective effects of topiroxostat, a selective xanthine oxidoreductase inhibitor, in patients with diabetic nephropathy and hyperuricemia: a randomized, double-blind, placebo-controlled, parallel-group study (UPWARD study). Clin Exp Nephrol 22: 860870. [Crossref]

9. Mizukoshi T, Koto S, Ando M, Sobajima H, Ohashi N, et al. (2018) Renoprotective effects of topiroxostat for hyperuremic patients with overt diabetic nephropathy study (ETUDE Study): A prospective, randomized, multicenter clinical trial. Nephrology 23: 1023-1030.

10. Kosugi T, Nakayama T, Heinig M, Zhang L, Yuzawa Y, et al. (2009) Effect of lowering uric acid on renal disease in the type 2 diabetic $\mathrm{db} / \mathrm{db}$ mice. Am J Physiol Renal Physiol 297: F481-488. [Crossref] 
Nakamura T (2019) The influence of albumin on the plasma xanthine oxidoreductase inhibitory activity of allopurinol, febuxostat and topiroxostat: Insight into extra-urate lowering effect

11. Lee HJ, Jeong KH, Kim YG, Moon JY, Lee SH, et al. (2014) Febuxostat ameliorates diabetic renal injury in a streptozotocin-induced diabetic rat model. Am J Nephrol 40: $56-63$.

12. Nakamura T, Murase T, Nampei M, Morimoto N, Ashizawa N, et al. (2016) Effects of topiroxostat and febuxostat on urinary albumin excretion and plasma xanthine oxidoreductase activity in db/db mice. Eur J Pharmacol 780: 224-231.

13. Sezai A, Obata K, Abe K, Kanno S, Sekino H (2017) Cross-Over Trial of Febuxosta and Topiroxostat for Hyperuricemia With Cardiovascular Disease (TROFEO Trial). Circ J 81: 1707-1712. [Crossref]

14. Terawaki H, Hoshi H, Kazama JJ (2017) Effect of switching xanthine oxidoreductase inhibitor from febuxostat to topiroxostat on urinary protein excretion. Clin Exp Nephrol 21: 356-357. [Crossref]

15. Fasano M, Curry S, Terreno E, Galliano M, Fanali G, et al. (2005) The extraordinary ligand binding properties of human serum albumin. IUBMB Life 57: 787-796. [Crossref]

16. Murase T, Nampei M, Oka M, Miyachi A, Nakamura T (2016) A highly sensitive assay for xanthine oxidoreductase activity using a combination of [13C2,15N2] xanthine and liquid chromatography/triple quadrupole mass spectrometry. J Labelled Comp Radiopharm 59: 214-20.

17. Murase T, Nampei M, Oka M, Miyachi A, Nakamura T (2016) A highly sensitive assay of human plasma xanthine oxidoreductase activity using stable isotope-labeled xanthine and LC/TQMS. J Chromatogr B Analyt Technol Biomed Life Sci 1039: 51-58. [Crossref]

18. Klotz IM (1953) The proteins part B, Academic Press, New York, USA.

19. Hosoya T, Ohno I (2011) A repeated oral administration study of febuxostat (TMX-67), a non-purine-selective inhibitor of xanthine oxidase, in patients with impaired renal function in japan. $J$ Clin Rheumatol 4: S27.

20. Report of drug approval statement for topiroxostat, URIADEC® (in English), Summary of clinical pharmacology, Phase I repeated dose study.

21. Upton RN, Mould DR (2014) Basic concepts in population modeling, simulation, and model-based drug development: part 3-introduction to pharmacodynamic modeling methods. CPT Pharmacometrics Syst Pharmacol 3: e88. [Crossref]

22. Battelli MG, Bolognesi A, Polito L (2014) Pathophysiology of circulating xanthine oxidoreductase: new emerging roles for a multi-tasking enzyme. Biochim Biophys Acto 1842: 1502-1517.

23. Furuhashi M, Matsumoto M, Tanaka M, Moniwa N, Murasse T, et al., (2018) Plasma xanthine oxidoreductase activity as a novel biomarler of metabolic disorders in a general population. Circ J 82: 1892-1899.

24. Washino K, Kusunoki Y, Murase T, Nakamura T, Osugi K, et al., (2017) Xanthine oxidoreductase activity is correlated with insulin resistance and subclinical inflammation in young humans. Metabolism 70: 51-56.

25. Fujimura Y, Yamauchi Y, Murase T, Nakamura T, Fujita SI, et al. (2017) Relationship between plasma xanthine oxidoreductase activity and left ventricular ejection fraction and hypertrophy among cardiac patients. PLoS One 12: e0182699. [Crossref]

26. Hille R (1996) The Mononuclear Molybdenum Enzymes. Chem Rev 96: 2757-2816. [Crossref]

27. Massey V, Komai H, Palmer G, Elion GB (1970) On the mechanism of inactivation of xanthine oxidase by allopurinol and other pyrazolo[3,4-d]pyrimidines. $J$ Biol Chem 245: 2837-2844. [Crossref]

28. Matsumoto K, Okamoto K, Ashizawa N, Nishino T (2011) FYX-051: a novel and potent hybrid-type inhibitor of xanthine oxidoreductase. J Pharmacol Exp Ther 336: 95-103. [Crossref]

29. Vuignier K, Schappler J, Veuthey JL, Carrupt PA, Martel S (2010) Drug-protein binding: a critical review of analytical tools. Anal Bioanal Chem 398: 53-66. [Crossref]

30. Okamoto K, Eger BT, Nishino T, Kondo S, Pai EF, et al. (2003) An extremely potent inhibitor of xanthine oxidoreductase: Crystal structure of the enzyme-inhibitor complex and mechanism of inhibition. $J$ Biol Chem 278: 1848-1855.
31. Nishino T, Okamoto K, Ege BT, Pai EF, Nishino T (2008) Mammalian xanthine oxidoreductase-mechanism of transition from xanthine dehydrogenase to xanthine oxidase. FEBS J 275: 3278-3289.

32. Okamoto K, Matsumoto K, Hille R, Eger BT, Pai EF, et al., (2004) The crystal structure of xanthine oxidoreductase during catalysis: Implications for reaction mechanism and enzyme inhibition. Proc Natl Acad USA 101: 7931-7936.

33. Tam HK, Kelly AS, Metzig AM, Steinberger J, Johnson LA (2014) Xanthine oxidase and cardiovascular risk in obese children. Child Obes 10: 175-180. [Crossref]

34. Tam HK (2016) Weight loss mediated reduction in xanthine oxidase activity and uric acid clearance in adolescents with severe obesity. Child Obes 12: 286-291.

35. Desco MC, Asensi M, Marquez R, Martinez JV, Vento M, et al. (2002) Xanthine oxidase is involved in free radical production in type 1 diabetes: protection by allopurinol. Diabetes 51: 1118-1124.

36. Kuppusamy UR, Indran M, Rokiah P (2005) Glycaemic control in relation to xanthine oxidase and antioxidant indices in Malaysian Type 2 diabetes patients. Diabet Med 22 1343-1346. [Crossref]

37. Miric DJ, Kisic BM, Danic SF, Grbic R, Dragojevic I, et al. (2016) Xanthine Oxidase Activity in Type 2 Diabetes Mellitus Patients with and without Diabetic Periphera Neuropathy. J Diabetes Res 4370490. [Crossref]

38. Spiekermann S, Landmesser U, Dikalvo S, Bredt M, Gamez G, et al. (2003) Electron spin resonance characterization of vascular xanthine and $\mathrm{NAD}(\mathrm{P}) \mathrm{H}$ oxidase activity in patients with coronary artery disease: Relation to endothelium-dependent vasodilation. Circulation 107: 1383-1389.

39. Landmesser U, Spiekermann S, Dikalov S, Tatge H, Wilke R, et al. (2002) Vascula oxidative stress and endothelial dysfunction in patients with chronic heart failure: Role of xanthine-oxidase and extracellular superoxide dismutase. Circulation 106: 3073 3078. [Crossref]

40. Majkic-Singh N, Bogavac L, Kalimanovska V, Jelic X, Spasic S, et al., (1987) Spectrophotometric assay of xanthine oxidase with 2,2'-azino-di(3-ethylbenzthiazoline6-sulphonate) (ABTS) as chromogen. Clin Chim Acta 162: 29-36.

41. Shamma'a MH, Nasrallah SM, al-Khalidi UA (1973) Serum xanthine oxidase. An experience with 2000 patients. Am J Dig Dis 18: 15-22. [Crossref]

42. McHale A, Grimes H, Coughlan MP, (1979) Human serum xanthine oxidase: fluorometric assay applicable to the investigation of liver disorders. Int J Biochem 10 : 317-319.

43. Yamamoto T, Moriwaki Y, Takahashi S, Tsutsumi Z, Yamakita J, et al. (1996) Determination of human plasma xanthine oxidase activity by high-performance liquid chromatography. J Chromatogr B 681: 395-400.

44. Kanabrocki EL, Third JL, Ryan MD, Nemchausky BA, Shirazi P, et al. (2000) Circadian relationship of serum uric acid and nitric oxide. JAMA 283: 2240-2241. [Crossref]

45. Srinivasan B, Rebecca F, Bin W, Gou W, Xiaorui C, et.al., (2016) A Randomized controlled trial of the effects of febuxostat therapy on adipokaines and, Canadian journal of kidney health and disease, 3: 1-11.

46. Della CE, Stirpe F (1968) The regulation of rat-liver xanthine oxidase: Activation by proteolytic enzymes. FEBS Lett 2: 83-84.

47. Stirpe F, Della CE (1969) The regulation of rat liver xanthine oxidase. Conversion in vitro of the enzyme activity from dehydrogenase (type D) to oxidase (type O). $J$ Biol Chem 244: 3855-3863.

48. Nishino T (1994) The conversion of xanthine dehydrogenase to xanthine oxidase and the role of the enzyme in reperfusion injury. J Biochem 116: 1-6. [Crossref]

49. Barr JT, Choughle KV, Nepal S, Wong T, Chaudhry AS, et al. (2014) Why do most human liver cytosol preparations lack xanthine oxidase activity? Drug Metab Dispos 42: 695-699.

Copyright: (C2019 Nakamura T. This is an open-access article distributed under the terms of the Creative Commons Attribution License, which permits unrestricted use, distribution, and reproduction in any medium, provided the original author and source are credited. 